\title{
PLANEJAMENTO PARA AQUISIÇÃO E DOWNLOAD DE DADOS ESPACIAIS: ANÁLISE DA METODOLOGIA
}

\author{
Maria José Pinto \\ Instituto de Estudos Avançados (IEAv) \\ Trevo Cel Av José A. A. do Amarante, nº 1, Putim, 12228-001 - São José dos Campos - SP \\ maju@ieav.cta.br \\ Mônica Maria De Marchi \\ Instituto de Estudos Avançados (IEAv) \\ Trevo Cel Av José A. A. do Amarante, nº 1, Putim, 12228-001 - São José dos Campos - SP \\ monica@ieav.cta.br \\ Ana Cláudia Iwazaki \\ Instituto de Estudos Avançados (IEAv) \\ Trevo Cel Av José A. A. do Amarante, nº 1, Putim, 12228-001 - São José dos Campos - SP \\ anaclaudia@ieav.cta.br
}

\section{RESUMO}

Buscando contribuir com as pesquisas na área espacial uma metodologia foi proposta anteriormente para gerar um planejamento para uma constelação de satélites para adquirir e fazer o download de dados para as estações de solo, visando monitorar locais de interesse (alvos) com diferentes prioridades, dado um horizonte de planejamento. Neste trabalho, algumas situações na prática serão apresentadas para mostrar como a metodologia pode auxiliar no processo de tomada de decisão permitindo algumas análises automáticas que seriam difíceis de serem realizadas sem o seu auxílio, mesmo para instâncias pequenas. Por outro lado, outras situações serão apresentadas para mostrar que o modelo ainda precisa de novas adaptações para permitir uma aplicação efetiva na prática.

Palavras-chave: Sistemas Espaciais; Apoio à Decisão; Aquisição de Dados Espaciais; Modelo Matemático.

\begin{abstract}
Contributing to the space research, a methodology has been proposed in previous work to generate a scheduling for a constellation of satellites to acquire and download data to ground stations to monitor areas of interest (targets) with different priorities for a given planning horizon. In this paper, some practical situations are presented to show how the methodology can support the decision making process through automatic evaluations and how it would be difficult to perform them without the proposed methodology, even for small instances. On the other hand, other situations are presented showing some improvements necessary for an effective application of the mathematical model.
\end{abstract}

Keywords: Space Systems; Decision Support; Acquisition of Spatial Data; Mathematical Model. 


\section{Como Citar:}

PINTO, M.J.; DE MARCHI, M.M.; YWAZAKI, A.C. Planejamento para Aquisição e Download de Dados Espaciais: Análise da Metodologia. In: SIMPÓSIO DE PESQUISA OPERACIONAL E LOGÍSTICA DA MARINHA, 19., 2019, Rio de Janeiro, RJ. Anais [...]. Rio de Janeiro: Centro de Análises de Sistemas Navais, 2019.

\section{INTRODUÇÃO}

O Programa Nacional de Atividades Espaciais (PNAE) (AEB, 2012) busca enfatizar suas prioridades em relação às atividades espaciais e seu interesse estratégico no domínio das tecnologias espaciais visando fortalecer a autonomia e a soberania do país. Adicionalmente, a riqueza do país em recursos naturais torna fundamental o desenvolvimento de tecnologias que auxiliem em um monitoramento eficiente e eficaz do território brasileiro. Essa necessidade vem de encontro com a solicitação da Estratégia Nacional de Defesa (END) de uma maior capacidade de monitoramento do território e das fronteiras do nosso país (MINISTÉRIO DA DEFESA, 2012). Desta forma, pesquisas que busquem um melhor gerenciamento dos sistemas espaciais são essenciais para apoiar o processo de tomada de decisão em diversos cenários de aplicação, tanto no âmbito da defesa como no meio civil.

Neste contexto, um trabalho de pesquisa está sendo realizado visando otimizar o emprego dos sistemas espaciais. Especificamente, um modelo matemático foi proposto em (PINTO et al., 2018) para gerar o planejamento para aquisição e download de dados para uma constelação de satélites e de estações de solo, com foco no monitoramento de áreas de interesse (alvos) que possuem prioridades diferentes, dentro de um horizonte de planejamento. Este modelo busca atender restrições de natureza operacional referentes às janelas de visibilidade (oportunidades de contato entre o satélite e o alvo/estação de solo) e à capacidade de tempo de processamento, além de modelar requisitos do cenário, definidos pelo usuário do sistema espacial, como o intervalo mínimo de tempo entre observações sucessivas de um mesmo alvo (Revisit Time - RT) ou o prazo máximo para que os dados coletados estejam disponíveis (Due Time - DT).

Novas restrições foram adicionadas a este modelo para permitir determinadas aplicações na prática (PINTO e DE MARCHI, 2019; PINTO et al., 2019). Em Pinto e De Marchi (2019), foi considerado o tempo de transição quando aquisições consecutivas de um mesmo satélite necessitarem de diferentes modos de aquisição pois, de acordo com Liang (2017), a troca de um modo de aquisição para outro não é automática e nenhum dado pode ser adquirido durante este intervalo de tempo. Desta forma, se existirem alvos próximos que possam ser adquiridos com modos de aquisição diferentes, um tempo de transição deverá ser levado em consideração evitando assim gerar uma solução infactível na prática. Em Pinto et al. (2019), a capacidade de armazenamento dos satélites foi modelada pois, dependendo do volume de dados adquiridos é importante considerar a capacidade de armazenamento, a qual pode permitir que novos dados sejam adquiridos considerando que espaços de armazenamento são liberados à medida que os dados já coletados são descarregados para as estações de solo. Em ambos os trabalhos, o modelo com as novas restrições foi aplicado em um exemplo ilustrativo para mostrar a necessidade e relevância de incluir tanto o tempo de transição quanto as restrições de armazenamento ao modelo.

De nosso conhecimento, são poucos os trabalhos que tratam este problema de forma integrada, ou seja, considerando tanto a aquisição dos dados dos alvos quanto a transferência dos dados coletados para as estações de solo (WANG E REINELT, 2011; WANG et al., 2011; KIM E CHANG, 2015; CHO et al., 2018). E, nestes trabalhos, nem sempre um 
modelo matemático foi apresentado para o problema ou as restrições para tratar RT e DT foram consideradas.

O presente trabalho apresenta algumas análises para mostrar como a metodologia desenvolvida até o momento pode auxiliar de forma efetiva na tomada de decisão em determinadas situações práticas. Além disso, outras situações práticas serão apresentadas para mostrar que o modelo ainda não contempla algumas restrições do problema. O resultado destas análises será apresentado nas Seções 2 e 3, respectivamente. Na Seção 4, algumas considerações finais são também apresentadas.

\section{RELEVÂNCIA DA METODOLOGIA NA PRÁTICA}

As análises apresentadas nesta seção estão baseadas no exemplo ilustrativo apresentado nos trabalhos anteriores (PINTO e DE MARCHI, 2019; PINTO et al., 2019). Neste exemplo, considera-se um horizonte de planejamento de três dias, três alvos (T1, T2 e T3), dois satélites (S1, S2) e uma estação de solo (ES). Considera-se ainda que, no período de planejamento, é possível observar 5 vezes o alvo T1; 2 vezes o alvo T2 e 4 vezes o alvo T3, resultando num total de 11 janelas de visibilidade para aquisição (A1 a A11). Além disso, os satélites S1 e S2 terão contato com a estação de solo 3 vezes durante o período, resultando num total de 6 janelas de visibilidade para download (D1 a D6). Estas janelas são apresentadas nas Tabelas 1 e 2, respectivamente, onde as colunas "Início" e "Fim" representam o momento inicial e final da janela de visibilidade (em minutos), dentro do horizonte de planejamento de 3 dias (que corresponde a um total de 4320 minutos) e a coluna "Duração" representa o tempo total (em minutos) de cada janela. Neste exemplo, a estação de solo somente pode atender um satélite por vez para realização de download.

Tabela 1 - Janelas de visibilidade para aquisição de dados para cada satélite.

\begin{tabular}{|c|c|c|c|c|c|}
\hline $\begin{array}{c}\text { Janelas de } \\
\text { Aquisiçãa }\end{array}$ & Satélite & Alvo & Início & Fim & Duração \\
\hline $\mathrm{A} 1$ & \multirow{6}{*}{$\mathrm{S} 1$} & $\mathrm{~T} 2$ & 1005 & 1009 & 4 \\
\hline $\mathrm{A} 2$ & & T3 & 1015 & 1019 & 4 \\
\hline A3 & & T1 & 1830 & 1834 & 4 \\
\hline A4 & & $\mathrm{T} 2$ & 2455 & 2461 & 6 \\
\hline A5 & & $\mathrm{T} 1$ & 3298 & 3302 & 4 \\
\hline A6 & & T3 & 3895 & 3900 & 5 \\
\hline A7 & \multirow{5}{*}{ S2 } & $\mathrm{T} 1$ & 624 & 629 & 5 \\
\hline A8 & & $\mathrm{T} 3$ & 874 & 877 & 3 \\
\hline A9 & & $\mathrm{T} 1$ & 2116 & 2121 & 5 \\
\hline A10 & & $\mathrm{T} 1$ & 3583 & 3588 & 5 \\
\hline A11 & & T3 & 3780 & 3783 & 3 \\
\hline
\end{tabular}

Tabela 2 - Janelas de visibilidade para download de dados para a estação de solo (ES).

\begin{tabular}{|c|c|c|c|c|}
\hline $\begin{array}{l}\text { Janelas de } \\
\text { Download }\end{array}$ & Satélite & Início & Fim & Duração \\
\hline D1 & \multirow{3}{*}{ S1 } & 1437 & 1442 & 5 \\
\hline D2 & & 2880 & 2884 & 4 \\
\hline D3 & & 4317 & 4320 & 3 \\
\hline D4 & \multirow{3}{*}{ S2 } & 1440 & 1444 & 4 \\
\hline D5 & & 2858 & 2865 & 7 \\
\hline D6 & & 4315 & 4319 & 4 \\
\hline
\end{tabular}


Para um melhor entendimento das análises e para facilitar a interpretação, o histograma de cobertura das janelas de visibilidade de aquisição e de download é apresentado na Fig. 1.

Figura 1 - Histograma de cobertura do exemplo ilustrativo para representar as janelas de visibilidade para aquisição (A1 a A11) e para download (D1 a D6).
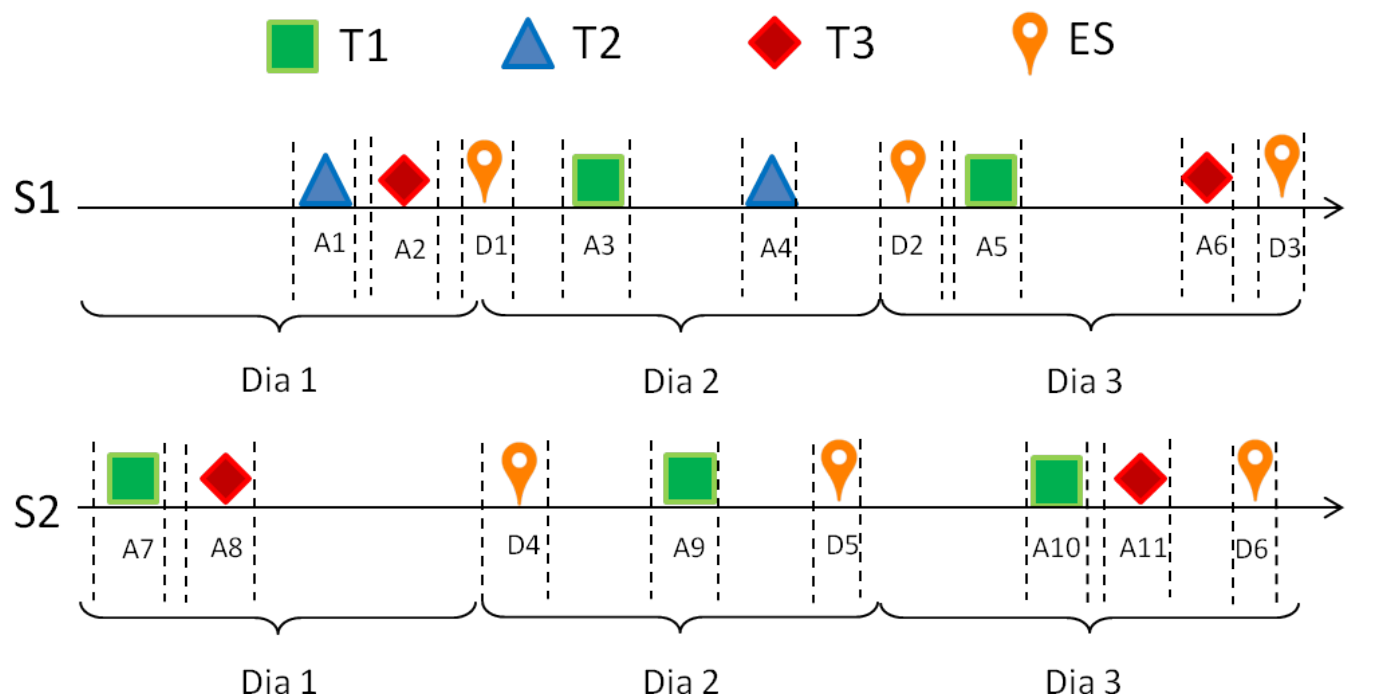

Fonte: Autores

A Tabela 3 apresenta os valores para RT e DT, para cada alvo. No caso, os valores de RT apresentados na tabela indicam de quanto em quanto tempo o alvo precisa ser visitado. Por exemplo, para o alvo T2 será de 3 em 3 dias $(\mathrm{RT}=3)$ e, para o alvo T3, será de 2 em 2 dias $(\mathrm{RT}=2)$. O valor de RT e o total de dias do horizonte de planejamento definem o máximo de vezes que será possível adquirir os dados de cada alvo (indicado na coluna $\mathbf{A}$ da Tabela 3). O valor de DT indica o tempo máximo (em minutos), dentro do horizonte de planejamento de 3 dias, em que os dados coletados precisam ser descarregados para a estação de solo (ES). Para os alvos que precisam ser observados mais de uma vez durante o horizonte de planejamento, serão definidos valores de DT para cada uma das observações.

Tabela 3 - Revisit Time (RT) e Due Time (DT) para cada alvo.

\begin{tabular}{|c|c|c|c|c|}
\hline Alvo & RT & A & Observação & DT \\
\hline \multirow{3}{*}{$\mathrm{T} 1$} & \multirow{3}{*}{1} & \multirow{3}{*}{3} & $1^{\underline{\mathrm{a}}}$ & Início do segundo dia (1600 min) \\
\hline & & & $2^{\underline{0}}$ & Final do segundo dia (2880 $\mathrm{min})$ \\
\hline & & & $3^{-0}$ & Final do terceiro dia (4320 min) \\
\hline $\mathrm{T} 2$ & 3 & 1 & $1^{\mathrm{a}}$ & Final do segundo dia (2880 $\mathrm{min})$ \\
\hline \multirow{2}{*}{$\mathrm{T} 3$} & \multirow{2}{*}{2} & \multirow{2}{*}{2} & $1^{\mathrm{a}}$ & Início do segundo dia (1500 min) \\
\hline & & & $2^{-}$ & Final do terceiro dia (4320 min) \\
\hline
\end{tabular}

Como utilizaremos esta informação nas análises que serão apresentadas a seguir, é importante destacar que estamos assumindo que o valor de DT apresentado na Tabela 3 não está contabilizando o tempo necessário para que a informação esteja efetivamente disponível para o usuário final, após o descarregamento destes dados para a estação de solo. Este tempo inclui, por exemplo, o tempo para que os dados coletados estejam disponíveis no centro de 
processamento de dados e o tempo para o processamento destes dados. Por exemplo, se este tempo for de 1 hora (60 minutos) para o alvo T1 e o usuário solicitou a segunda aquisição deste alvo para as primeiras horas do terceiro dia do planejamento, o modelo matemático considerará que os dados deste alvo precisam ser descarregados para uma estação de solo até, no máximo, no final do segundo dia de planejamento, como apresentado na Tabela 3.

O exemplo considera ainda que será necessário um tempo de 1 minuto para processar os dados dos alvos para ambos os satélites e um tempo de 0,5 minutos para fazer o download dos dados, independente de qual satélite tenha adquirido os dados do alvo. Como apresentado em Pinto e De Marchi (2019) e Pinto et al. (2019), o modelo proposto foi implementado no AMPL (AMPL, 2019), gerando o planejamento apresentado na Tabela 4.

Tabela 4 - Solução do modelo para o exemplo ilustrativo.

\begin{tabular}{|c|c|c|c|c|}
\hline Alvo & Observação & Satélite & $\begin{array}{l}\text { Janela de } \\
\text { Aquisição }\end{array}$ & $\begin{array}{l}\text { Janela de } \\
\text { Download }\end{array}$ \\
\hline \multirow{3}{*}{$\mathrm{T} 1$} & $1^{\mathrm{a}}$ & $\mathrm{S} 2$ & A7 & D4 \\
\hline & $2^{-}$ & S2 & A9 & D5 \\
\hline & $3^{-0}$ & S2 & A10 & D6 \\
\hline $\mathrm{T} 2$ & $1^{\mathrm{a}}$ & S1 & A1 & D1 \\
\hline \multirow{2}{*}{$\mathrm{T} 3$} & $1^{\mathrm{a}}$ & S1 & $\mathrm{A} 2$ & D1 \\
\hline & $2^{-}$ & S1 & A6 & D3 \\
\hline
\end{tabular}

Algumas análises foram feitas e serão apresentadas em seguida, sendo que alguns dados de entrada do modelo (como capacidade de armazenamento e prioridade) serão apresentados à medida que forem necessários.

\subsection{ANÁLISE 1}

Pelo histograma da Fig. 1 é possível observar que o alvo T2 e a primeira aquisição do alvo T3 somente poderiam ser descarregados utilizando a janela de download D1 pois é a única janela que permite atender o Due Time destes alvos (veja Tabela 3). Mas, para isto, os alvos precisam ser adquiridos usando as janelas A1 e A2, como mostrou a solução apresentada na Tabela 4. Esta solução é possível pois assumimos que o download de dados para a estação de solo é feito em pacotes e, desta forma, os dados de alvos diferentes podem ser descarregados simultaneamente utilizando a mesma estação de solo desde que respeitem o tamanho da janela de visibilidade para adquirir os dados de ambos os alvos, que é o caso.

Em Pinto et al. (2019), os autores mostraram que, se ambos os satélites tiverem uma capacidade de armazenamento (CA) de 100 Mbits e que os alvos T1, T2 e T3 possuem, respectivamente, um volume de dados de 10, 20 e 30 Mbits, a mesma solução da Tabela 4 será gerada mostrando que a capacidade de 100 Mbits não excede a capacidade de armazenamento dos satélites. Entretanto, como também foi mostrado em Pinto et al. (2019), se reduzirmos a capacidade de armazenamento para 40 Mbits, os dados dos alvos T2 e T3 não podem mais ser adquiridos na sequência pois a capacidade de armazenamento do satélite $\mathrm{S} 1$ seria excedida (o volume total de dados seria de $50 \mathrm{Mbits}$ ).

Se considerarmos que todos os alvos tem prioridade igual a 1 e, como somente uma das aquisições poderá ser feita, o AMPL define que a demanda do alvo T2 não será atendida, conforme apresentado na Tabela 5 (esta mesma solução seria obtida se o alvo T2 fosse menos prioritário em relação ao alvo T3). 
Tabela 5 - Solução do modelo para CA = 40Mbit.

\begin{tabular}{|c|c|c|c|c|}
\hline Alvo & Observação & Satélite & $\begin{array}{c}\text { Janela de } \\
\text { Aquisição }\end{array}$ & $\begin{array}{c}\text { Janela de } \\
\text { Download }\end{array}$ \\
\hline \multirow{3}{*}{ T1 } & $1^{\text {a }}$ & S2 & A7 & D4 \\
\cline { 2 - 5 } & $2^{-\underline{0}}$ & S2 & A9 & D5 \\
\cline { 2 - 5 } & $3^{-}$ & S2 & A10 & D6 \\
\hline \multirow{2}{*}{ T3 } & $1^{\underline{a}}$ & S1 & A2 & D1 \\
\cline { 2 - 5 } & $2^{\underline{0}}$ & S1 & A6 & D3 \\
\hline
\end{tabular}

Entretanto, se considerarmos que a obtenção do alvo T2 passa a ter prioridade em relação ao alvo T3 ( $\left.\mathrm{p}_{\mathrm{T} 2}>\mathrm{p}_{\mathrm{T} 3}\right)$, a solução indicaria o atendimento do alvo T2 e, com isto, somente uma aquisição do alvo T3 é possível, como mostra a Tabela 6.

Tabela 6 - Solução do modelo para $\mathrm{CA}=40 \mathrm{Mbit}$ e $\mathrm{p}_{\mathrm{T} 2}>\mathrm{p}_{\mathrm{T} 3}$.

\begin{tabular}{|c|c|c|c|c|}
\hline Alvo & Observação & Satélite & $\begin{array}{l}\text { Janela de } \\
\text { Aquisição }\end{array}$ & $\begin{array}{l}\text { Janela de } \\
\text { Download }\end{array}$ \\
\hline \multirow{3}{*}{$\mathrm{T} 1$} & $1^{\mathrm{a}}$ & S2 & A7 & D4 \\
\hline & $2^{-}$ & S2 & A9 & D5 \\
\hline & $3^{-}$ & S2 & A10 & D6 \\
\hline $\mathrm{T} 2$ & $1^{\mathrm{a}}$ & $\mathrm{S} 1$ & A1 & D1 \\
\hline T3 & $2^{-}$ & S1 & A6 & D3 \\
\hline
\end{tabular}

Esta solução, em determinadas situações práticas, pode não ser satisfatória do ponto de vista do decisor. Considere, por exemplo, que o alvo T3, apesar de possuir prioridade menor que o alvo T2, tenha sido solicitado para realização de um monitoramento para detecção precoce de um cenário que permita ações de mitigação para evitar que uma crise ocorra. Neste caso, adquirir somente uma vez o dado e, ainda, somente no final do horizonte de planejamento pode não ser suficiente pois o decisor gostaria de ter diferentes dados disponíveis de um mesmo alvo para detectar possíveis diferenças durante o horizonte de planejamento.

Esta análise mostra a influência da definição de prioridades e da capacidade de armazenamento dos satélites.

\subsection{ANÁLISE 2}

Observe que, ao reduzir a capacidade de armazenamento para 40Mbit na análise anterior, o planejamento não contemplou o atendimento do alvo T2 (veja Tabela 5) pois, como já observado anteriormente, o alvo T2 e a primeira aquisição do alvo T3 somente poderiam ser descarregados utilizando a janela de download D1 por ser a única janela que permite atender o Due Time destes alvos. Mas, observe que o modelo não indicou a aquisição do alvo T3 pelo segundo satélite utilizando a janela A8 pois seu início ocorre apenas 37 minutos após o Due Time da primeira aquisição do alvo T3 (a janela D4 inicia no tempo 1537 e o Due Time da primeira aquisição do alvo T3 é 1500). Caso o valor de DT para a primeira aquisição do alvo T3 $\left(\mathrm{DT}_{31}\right)$ pudesse ser relaxado um pouco passando a ser $1550\left(\mathrm{DT}_{31}=1550\right)$, seria possível atender toda a demanda, pois a solução obtida pelo AMPL seria a solução apresentada na Tabela 7. Agora, os dados da primeira aquisição do alvo T3 
podem ser adquiridos usando a janela $\mathrm{A} 8$ e descarregados usando a janela $\mathrm{D} 4$, permitindo que o alvo T2 seja novamente descarregado utilizando a janela D1, como mostra a Tabela 7.

Tabela 7 - Solução do modelo para $\mathrm{CA}=40 \mathrm{Mbit}$ e $\mathrm{DT}_{31}=1550$.

\begin{tabular}{|c|c|c|c|c|}
\hline Alvo & Observação & Satélite & $\begin{array}{c}\text { Janela de } \\
\text { Aquisição }\end{array}$ & $\begin{array}{c}\text { Janela de } \\
\text { Download }\end{array}$ \\
\hline \multirow{3}{*}{ T1 } & 1 & $\mathrm{~S} 2$ & $\mathrm{~A} 7$ & $\mathrm{D} 4$ \\
\cline { 2 - 5 } & 2 & $\mathrm{~S} 2$ & $\mathrm{~A} 9$ & $\mathrm{D} 5$ \\
\cline { 2 - 5 } & 3 & $\mathrm{~S} 2$ & $\mathrm{~A} 10$ & $\mathrm{D} 6$ \\
\hline $\mathrm{T} 2$ & 1 & $\mathrm{~S} 1$ & $\mathrm{~A} 1$ & $\mathrm{D} 1$ \\
\hline \multirow{2}{*}{$\mathrm{T} 3$} & 1 & $\mathrm{~S} 1$ & $\mathrm{~A} 8$ & $\mathrm{D} 4$ \\
\cline { 2 - 5 } & 2 & $\mathrm{~S} 1$ & $\mathrm{~A} 6$ & $\mathrm{D} 3$ \\
\hline
\end{tabular}

Cabe observar que, da forma como o modelo está definido não é possível a identificação automática deste tipo de situação. Entretanto, uma possibilidade seria gerar o planejamento com os valores de entrada definidos inicialmente e, em caso de necessidade, gerar um novo planejamento relaxando alguns parâmetros para verificar se uma solução diferente é obtida e, principalmente, atende às necessidades do decisor.

$\mathrm{O}$ relaxamento de DT sugerido poderia ser definido pelo próprio decisor e solicitante da demanda ao ser informado que esta alteração de requisito poderia contemplar a obtenção de novos dados. Outra possibilidade para obter o resultado com mais dados, seria reduzir o tempo necessário para disponibilizar o dado final para o solicitante, após o descarregamento dos dados do alvo T3 na estação de solo pois, como comentado anteriormente, o valor de DT apresentado na Tabela 3 já está descontando este tempo. Neste caso, a análise poderia contribuir para identificar a necessidade de melhoria de métodos para realização do processamento dos dados.

A análise também mostra a influência da definição do Due Time no resultado final do planejamento.

\section{ADAPTAÇÕES DA METODOLOGIA PARA APLICAÇÃO PRÁTICA}

A seção anterior mostrou que a metodologia proposta pode auxiliar na análise automática de determinadas situações na prática. Entretanto, existem restrições do problema que o modelo ainda não atende e, com isto, adaptações serão necessárias para gerar um planejamento factível na prática.

\subsection{SOBREPOSIÇÃO DAS JANELAS DE VISIBILIDADE}

Pela Tabela 2, algumas janelas de visibilidade de download possuem um tempo de sobreposição (veja as janelas D1 e D4 e as janelas D3 e D6), ou seja, estamos considerando que é possível que a estação de solo esteja em contato com os dois satélites em determinados momentos. Esta situação é possível na prática e, pela literatura, uma estação de solo pode receber dados de diferentes satélites desde que o tempo dedicado a cada satélite seja respeitado.

O modelo atual não considera este tempo de dedicação e, com isto, a solução gerada pelo modelo pode ser infactível em determinadas situações. Por exemplo, aumentamos para 3 minutos o tempo para realização do download dos dados e o modelo gera a mesma solução apresentada na Tabela 4, onde todos os downloads são realizados logo no início das janelas 
de download. A Tabela 8 reapresenta a solução da Tabela 4 incluindo o momento inicial definido pelo modelo (valores entre parênteses). Mas, se forem necessários 3 minutos para fazer o download dos alvos, a primeira aquisição do alvo T1 e a segunda aquisição do alvo T3 seriam descarregadas 1 minuto mais tarde em relação à solução da Tabela 8 (momento 1441 para o alvo T1 e momento 4318 para o alvo T3). E, neste caso, a janela D3 não poderia mais ser utilizada para adquirir a segunda aquisição do alvo T3, pois não haveria tempo hábil para a realização total do download que seria finalizado no momento 4321, que excede o tempo final desta janela.

Tabela 8 - Solução da Tabela 4 destacando o momento inicial de aquisição e download.

\begin{tabular}{|c|c|c|c|c|}
\hline Alvo & Observação & Satélite & $\begin{array}{c}\text { Janela de } \\
\text { Aquisição }\end{array}$ & $\begin{array}{c}\text { Janela de } \\
\text { Download }\end{array}$ \\
\hline \multirow{3}{*}{ T1 } & $1^{\text {a }}$ & S2 & A7 (624) & D4 (1440) \\
\cline { 2 - 5 } & $2^{\underline{o}}$ & S2 & A9 (2116) & D5 (2858) \\
\cline { 2 - 5 } & $3^{\text {o }}$ & S2 & A10 (3585) & D6 (4315) \\
\hline T2 & $1^{\text {a }}$ & S1 & A1 (1005) & D1 (1437) \\
\hline \multirow{2}{*}{ T3 } & $1^{\underline{a}}$ & S1 & A2 (1015) & D1 (1437) \\
\cline { 2 - 5 } & $2^{\underline{o}}$ & S1 & A6 (3895) & D3 (4317) \\
\hline
\end{tabular}

\subsection{AGILIDADE DO SENSOR}

Como comentado anteriormente, o tempo de transição foi modelado para o caso em que aquisições consecutivas de um mesmo satélite necessitam de diferentes modos de aquisição. Entretanto, o modelo ainda não considera o tempo de transição para movimentar o sensor entre a aquisição de diferentes alvos por um mesmo satélite.

Para ilustrar a importância de considerar este tempo de transição, considere no exemplo ilustrativo da Seção 2, que o tempo para adquirir os alvos T2 e T3 fosse agora de 3 minutos e que seria necessário um tempo de 10 minutos para que o sensor adquira o alvo T2 e, em seguida, direcione o sensor para adquirir o alvo T3, para o satélite S1. Neste caso, não seria possível utilizar a janela A2 para adquirir o alvo T3 pois sua aquisição poderia somente iniciar no tempo $1017(1005+2+10)$ e, somando o tempo de 3 minutos para adquirir o alvo T3, a aquisição somente seria finalizada no tempo 1020, que está acima do limite superior da janela A2, que é de 1019.

Para modelar a agilidade do sensor, serão avaliadas duas possibilidades: incluir uma restrição parecida com a restrição incluída em Pinto e De Marchi (2019) ou adaptar esta restrição para que contemple a aquisição de diferentes alvos (não somente de alvos próximos que possuem modos de aquisição diferentes) e inclua tanto o tempo para trocar de modo de aquisição quanto o tempo para ir de um alvo a outro.

\section{CONSIDERAÇÕES FINAIS}

Neste trabalho, o problema de planejamento para aquisição e download de dados por um sistema espacial composto por satélites e estações de solo foi abordado, onde algumas análises da metodologia proposta em trabalhos anteriores foram apresentadas, para mostrar como o modelo pode auxiliar na tomada de decisão na prática. Um exemplo ilustrativo foi utilizado para mostrar o resultado destas análises, permitindo também observar que, mesmo para uma instância pequena com poucos satélites, alvos e estações de solo, a visualização das situações descritas não é simples e que o uso do modelo matemático é 
necessário para auxiliar nestas análises. Esta necessidade ficará mais evidente à medida que o problema se tornar mais complexo, envolvendo muitos vetores.

Por outro lado, este trabalho também mostrou que existem restrições do problema que ainda não foram modeladas e que são fundamentais para permitir uma aplicação na prática. Desta forma, o próximo passo do trabalho será a modelagem destas restrições e a realização de testes computacionais para validação do novo modelo, utilizando instâncias maiores. Dependendo da complexidade, serão também desenvolvidas abordagens heurísticas para tratar o problema. Abordagens heurísticas também serão necessárias em situações onde um replanejamento for necessário. A inclusão de cenários que precisam de informações atualizadas constantemente para apoiar o processo de tomada de decisão, como em cenários de busca e salvamento, são situações que poderiam demandar replanejamentos. Por exemplo, alvos podem ser adicionados (se um novo ponto de busca for identificado) ou eliminados (se for verificado que estes alvos estão muito distantes da região de busca provável). Mesmo que nenhum alvo seja incluído/eliminado, alguns requisitos dos alvos podem ser atualizados durante o planejamento como, por exemplo, a resolução da imagem quando informações mais detalhadas tornam-se necessárias.

A metodologia desenvolvida pode auxiliar no processo de tomada de decisão independente do nível de decisão a ser tomada, sendo o decisor um usuário ou o proprietário do sistema espacial. Sob a perspectiva de um usuário que tenha à sua disposição diferentes fornecedores de produtos espaciais, onde cada fornecedor oferece uma lista de produtos com características específicas, o decisor pode utilizar a metodologia proposta para auxiliá-lo na decisão sobre qual o fornecedor poderá atender às suas necessidades em termos de tempo e características da sua demanda. Sob a perspectiva do proprietário do sistema, visando atender um conjunto de demandas de dados espaciais com características diferentes, a metodologia proposta pode auxiliar no planejamento para que a aquisição dos dados maximize o atendimento das demandas. Aqui cabe ressaltar que, até o momento, o trabalho abordou o valor da informação a ser obtida considerando parâmetros técnicos, mas não leva em consideração ainda o custo para obtenção de dados. Este fator pode influenciar na definição de prioridades e na definição de qual satélite será escolhido para atender uma determinada demanda.

\section{REFERÊNCIAS BIBLIOGRÁFICAS}

[1] AEB. Programa Nacional de Atividades Espaciais (PNAE): 2012-2021. Brasília (2012). Disponível em: http://portal-antigo.aeb.gov.br/wpcontent/uploads/2013/03/PNAE-Portugues.pdf. Acesso em: Setembro de 2019.

[2] MINISTÉRIO DA DEFESA. Política Nacional de Defesa: Estratégia Nacional de Defesa (END). Brasília (2012). Disponível em: https://www.defesa.gov.br/arquivos/estado_e_defesa/END-PND_Optimized.pdf. Acesso em: Setembro de 2019.

[3] PINTO, M. J.; BARROS, A. I.; NOOMEN, R.; VAN GELDER, P. H. A. J. M.; T. LAMBALLAIS TESSENSOHN, T. (2018). A new model proposal for integrated satellite constellation scheduling within a planning horizon given operational constraints. Proceedings of the 7th International Conference on Operations Research and Enterprise Systems (ICORES), p. 312-319, 2018. ISBN 978-989-758-285-1. DOI: $10.5220 / 0006655003120319$.

[4] PINTO, M. J.; DE MARCHI, M. M. (2019). Análise do Modelo Matemático Referente ao Sequenciamento para Aquisição e Download de Dados por uma 
Constelação de Satélites. In: LI Simpósio Brasileiro de Pesquisa Operacional (SBPO), Limeira, SP.

[5] LIANG, S. (2017). Comprehensive Remote Sensing. Elsevier. 3134 páginas. 2017.

[6] PINTO, M. J.; DE MARCHI, M. M.; BARROS, A. I. (2019). Adaptação do Modelo Matemático referente ao Planejamento para Aquisição e Download de Dados Espaciais. In: XXI Simpósio de Aplicações Operacionais em Áreas de Defesa (SIGE).

[7] WANG, P.; REINELT, G. (2011). Solving the earth observing satellite constellation scheduling problem by branch-and-price. Operations Research Proceedings 2010, Springer, Berlin, Heidelberg, p. 491-496, 2011.

[8] WANG, P.; REINELT, G.; GAO, P.; TAN, Y. (2011). A model, a heuristic and a decision support system to solve the scheduling problem of an earth observing satellite constellation. Computers \& Industrial Engineering, v. 61, p. 322-335.

[9] KIM, H.; CHANG, J. K. (2015). Mission scheduling optimization of SAR satellite constellation for minimizing system response time. Aerospace Science and Technology, v. 40, p. 17-32.

[10] CHO, D-H; KIM, J-H; CHOI, J-L; AHN, J. (2018). Optmization-based scheduling method for agile earth-observing satellite constellation. Journal of Aerospace Information Systems, v. 15, p. 611-626.

[11] AMPL. Streamlined modeling for real optmization. Disponível em: $<$ www.ampl.com>. Acesso em: Junho de 2019. 